\title{
Embarazo saludable
}

\author{
Healthy pregnancy
}

\author{
Rosa María Guevara Cabrera ${ }^{a}$, Anabel Antonio-Rivera ${ }^{b}$, Rosa Silvana Torres Guevara $^{c}$, \\ Rosario Barrera Gálvez ${ }^{d}$, Rocío Belem Mayorga-Ponce ${ }^{e}$, Rosa María Baltazar-Téllez ${ }^{f}$
}

\begin{abstract}
:
To improve the health of newborns it is important that mothers carry a healthy pregnancy during pregnancy. Normal pregnancy is the woman's physiological state that begins with fertilization and ends with childbirth and the birth of the newborn. The mother presents physical and physiological changes that she must know through her prenatal consultations to strengthen preventive actions during pregnancy for a happy term.
\end{abstract}

The World Health Organization (WHO) defines it as quality care during pregnancy, childbirth and the postnatal period, establishing recommendations on sustainable development objectives (SDS) that guarantee a healthy pregnancy, with the opportunity to identify some warning signs that may arise and complicate pregnancy such as: headache, tinnitus, phosphenes, edema of the lower limbs, bleeding, fever, transvaginal losses, contractions, seizures and dysuria.

There are also tools that support pregnancy care, such as NOM-007-SSA2-2016, For the care of women during pregnancy, delivery and puerperium, and for the newborn and the NOM-O34- SSA2-2013, For the prevention and control of birth defects. Guaranteeing healthy and risk-free motherhood for all Mexican women contributes to reducing maternal and perinatal morbidity and mortality.

Keywords:

Healthy pregnancy, prenatal consultations, pregnancy, prevention, risk factors

\section{Resumen:}

Para mejorar la salud de los recién nacidos es importante que las madres cursen un embarazo saludable durante la gestación. El embarazo normal es el estado fisiológico de la mujer que se inicia con la fecundación y termina con el parto y el nacimiento del recién nacido. La madre presenta cambios físicos y fisiológicos que debe conocer a través de sus consultas prenatales para fortalecer acciones preventivas durante el embarazo para un feliz término.

La Organización Mundial de la Salud (OMS) lo define como la atención de calidad durante el embarazo, el parto y el periodo postnatal., estableciendo recomendaciones en los objetivos de desarrollo sustentable (ODS)que garanticen un embarazo saludable, con la oportunidad de identificar algunos signos de alarma que puedan presentarse y complicar el embarazo como: cefalea, acufenos, fosfenos, edema de miembros inferiores, sangrado, fiebre, perdidas transvaginales, contracciones, convulsiones y disuria. También existen herramientas que sirven de apoyo para la atención del embarazo como lo es la NOM-007-SSA2-2016, Para la atención de la mujer durante el embarazo, parto y puerperio, y de la persona recién nacida y la NOM-O34-SSA2-2013, Para la prevención y control de los defectos al nacimiento. Garantizar una maternidad saludable y sin riesgos en todas las mujeres mexicanas, contribuye a disminuir la morbilidad y mortalidad materna y perinatal.

\section{Palabras Clave:}

Embarazo saludable, consultas prenatales, embarazo, prevención, factores de riesgo

\footnotetext{
a Autor de Correspondencia, Universidad Autónoma del Estado de Hidalgo, Instituto de Ciencias de la Salud ORCID: 0000-0002-13092747, Email: rosy_guevara29@hotmail.com

${ }^{\mathrm{b}}$ Hospital General, Pachuca de Soto, Hidalgo, Email: anyanriverita@gmail.com

Universidad Autónoma del Estado de Hidalgo, Instituto de Ciencias de la Salud ORCID: 0000-0002-5006-4745, Email: stkita3@hotmail.com

d Universidad Autónoma del Estado de Hidalgo, Instituto de Ciencias de la Salud, ORCID: 0000-0002-1949-5424, Email: rosario_barrera@uaeh.edu.mx

Universidad Autónoma del Estado de Hidalgo, Instituto de Ciencias de la Salud, ORCID: 0000-0003-3544-7171, Email: robel62@hotmail.com

Universidad Autónoma del Estado de Hidalgo, Instituto de Ciencias de la Salud, ORCID: 0000-002-2168-7564, Email: rbaltazartellez@yahoo.com.mx
} 


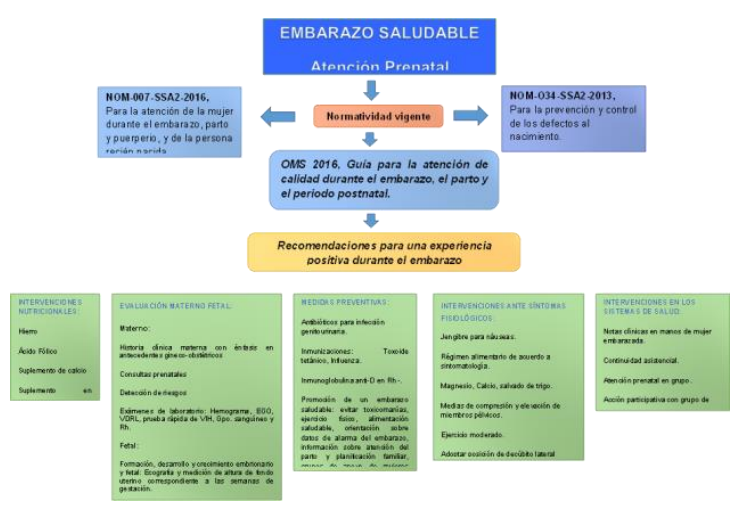

\section{Agradecimientos}

A la Universidad Autónoma del Estado de Hidalgo, Instituto de Ciencias de la Salud y al Área Académica de Enfermería, por las facilidades para llevar a cabo la publicación de este trabajo que sirve de guía a los profesionales de la salud, para enfatizar las acciones de un Embarazo Saludable y así poder disminuir la morbimortalidad materno infantil.

\section{Referencias}

[1] Secretaría de Salud (2001). Manual de atención. Embarazo Saludable Parto y Puerperio Seguros Recién Nacido Sano. https://www.paho.org/mex/index.php?option=com_docman\&view=do wnload\&alias=576-manual-de-atencion-embarazo-saludable-parto-ypuerperio-seguros-recien-nacido-sano\&category_slug=documentos-dereferencia\&Itemid=493Dilla T, Valladares A, Lizán L, Sacristán JA. Adherencia y persistencia terapéutica: causas, consecuencias y estrategias de mejora. Aten. Primaria 2009; 41(6): 342-48.

[2] OMS (2016) Recomendaciones de la OMS sobre atención prenatal para una experiencia positiva del embarazo. https://apps.who.int/iris/bitstream/handle/10665/250802/WHO-RHR-

$16.12-$

spa.pdf;jsessionid=65458955CDED9D551909124828338EB6?sequen $\mathrm{ce}=1$

[3] OPS (2011).Manual de atención Embarazo Saludable, Parto y Puerperio seguros, recién nacido sano. https://www.paho.org/mex/index.php?option=com_docman\&view=do cument\&layout=default\&alias $=576$-manual-de-atencion-embarazosaludable-parto-y-puerperio-seguros-recien-nacido-

sano\&category_slug=documentos-de-referencia\&Itemid=493 